\title{
GIANT SUBMUCOSAL GASTRIC LIPOMA - CASE REPORT
}

\author{
Maria-Gabriela Aniței ${ }^{1}$, I. Huțanu ${ }^{1}$, A. Gervescu ${ }^{1}$, Oana Simionescu ${ }^{1}$, \\ Nora Lefter $^{2}$, V. Scripcariu ${ }^{1}$ \\ 1) University of Medicine and Pharmacy "Gr.T. Popa" Iaşi, Romania \\ First Surgical Oncology Unit, Regional Institute of Oncology, Iaşi, Romania \\ 2) Municipal Hospital, Gastroenterology Unit, Gura Humorului, Romania
}

\begin{abstract}
GIANT SUBMUCOSAL GASTRIC LIPOMA - CASE REPORT (Abstract): Gastric lipomas are rare benign tumors, representing less than $1 \%$ of all gastric tumors. They often have a submucosal origin, but in rare cases could originate in the subserosa. We report the case of a 72 year-old male who presented dyspeptic syndrome and superior gastro-intestinal bleeding (melena). The upper gastrointestinal endoscopy revealed a large, almost stenotic, submucosal oval-shaped mass, with a diameter of $10 \mathrm{~cm}$ located in the gastric antrum, with an area of ulcerated gastric mucosa. The abdominal computer tomography revealed a homogeneous well-defined mass with negative densitometry values that corresponded to the fatty tissue. The tumor was enucleated through antrotomy, with an uneventful postoperative course. CONCLUSION: Although rare, the gastric lipoma can be a cause of gastrointestinal bleeding, mimicking a malignant tumor.
\end{abstract}

\section{KEY WORDS: GASTRIC LIPOMA; GASTROTOMY; TUMOR ENUCLEATION}

\author{
SHORT TITLE: Gastric lipoma
}

HOW TO CITE: Aniței MG, Huțanu I, Gervescu A, Simionescu O, Lefter N, Scripcariu V. Giant submucosal gastric lipoma - case report. Jurnalul de chirurgie (Iași). 2013; 9(2): 189-192. DOI: 10.7438/1584-9341-9-2-12.

\section{INTRODUCTION}

Gastrointestinal lipomas are rare benign tumors that may occur anywhere along the gut. Most of them are located in the colon, ileum and jejunum [1,2]. With only 220 cases reported in the literature [14], gastric lipomas are even more unusual, and they occur mostly in the antrum having a submucosal origin [5]. The imaging examination can be highly suggestive for the diagnosis, while the histological examination offers the final and certain diagnosis. Computed Tomography (CT scan) is the imaging examination of choice for obtaining a specific diagnosis of lipoma [6]. However, the tumor may sometimes undergo significant inflammatory changes leading to a difficult differential diagnostic with the well-differentiated liposarcoma.

\section{CASE REPORT}

We examined a 72 years old man with a 4 month history of dyspeptic disorder, fatigue and melena, and without significant weight loss. He was previously medically treated with i.v. fluids and haemostatics. The medical history was significant for hypertension, atrial fibrillation and heart failure stage II NYHA. The administered medication included warfarin, diuretics, clopidogrel, angiotesin II receptor blocker, calcium channels blockers and selective beta-blocker.

Physical examination reveals an obese patient $\left(\mathrm{BMI}=30.5 \mathrm{~kg} / \mathrm{m}^{2}\right)$, with a general good status, and slightly pale teguments. The abdomen was flat and soft, with mild tenderness in epigastrum and no palpable masses. 
Laboratory tests showed anemic syndrome (hemoglobin $8.8 \mathrm{mg} / \mathrm{dL}$, hematocrit $28.5 \%$ ); tumor markers showed no abnormalities (CEA and CA19-9 in normal range). Blood chemistry profile and coagulation samples were within normal limits.

The upper gastrointestinal endoscopy revealed a large, oval-shaped, submucosal stenosing tumor, located in the gastric antrum. An area of ulceration was identified in the center of the tumor. Many biopsies were obtained (from superficial and deep layers), but they only showed unspecific inflammation of the gastric mucosa.

The CT scan revealed a homogeneous oval-shaped, well defined soft tissue mass in the gastric antrum area, measuring approximately $5.3 \times 8.1 \times 6.2 \mathrm{~cm}$, with negative densitometry values (-70 and -120

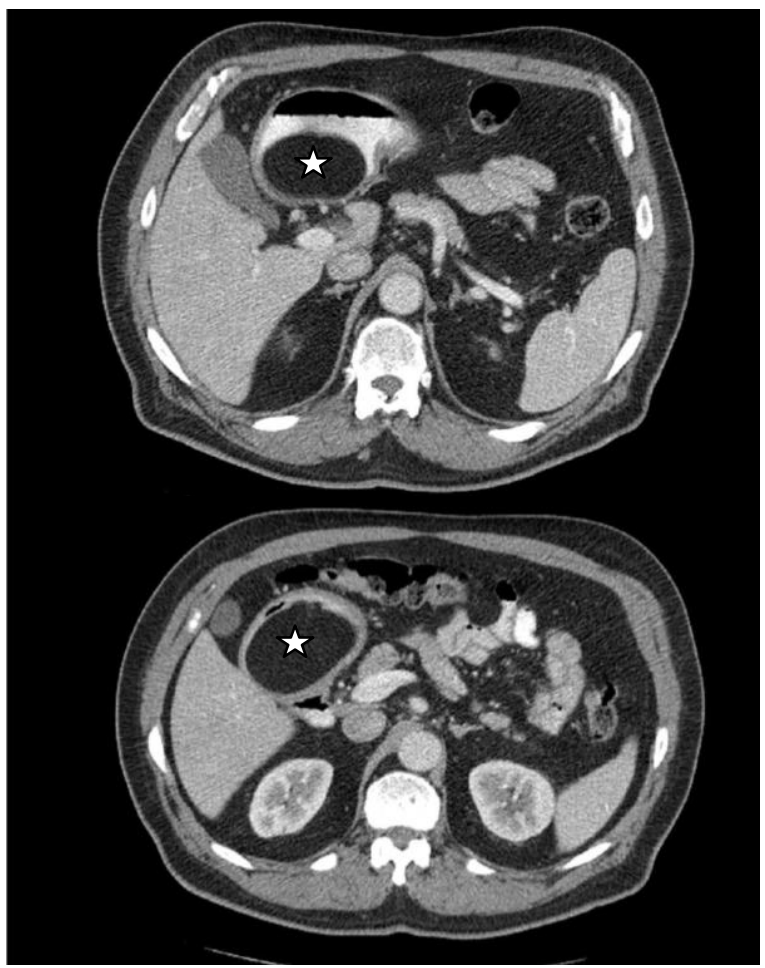

Fig. 1 CT-scan image showing a well-defined oval mass on the gastric antrum

An edge resection of the underlying layers, with endoluminal check of the gastric mucosa was performed, due to a $1 \mathrm{~cm} 2$ area of adhesion of the tumor to the gastric mucosa (Fig. 2, bottom).
Hounsfield units) that correspond to fatty tissue (Fig. 1), which on the barium study appeared as a smooth filling defect with a "bull's eye" appearance, slightly pressing on the duodenum.

The tumor was suspected to be a lipoma, a leiomyoma or a leiomyosarcoma.

The patient underwent midline supraumbilical laparotomy. The antropyloric region of the stomach reveals a yellowish, well defined tumor, with a longitudinal diameter of $8.5 \mathrm{~cm}$, placed in the posterior wall of gastric antrum, at a distance from the pyloric canal, without any other pathological findings.

The tumor was totally removed through an incision of gastric serosa and subserosa, dissected from the gastric mucosa and enucleated (Fig. 2).

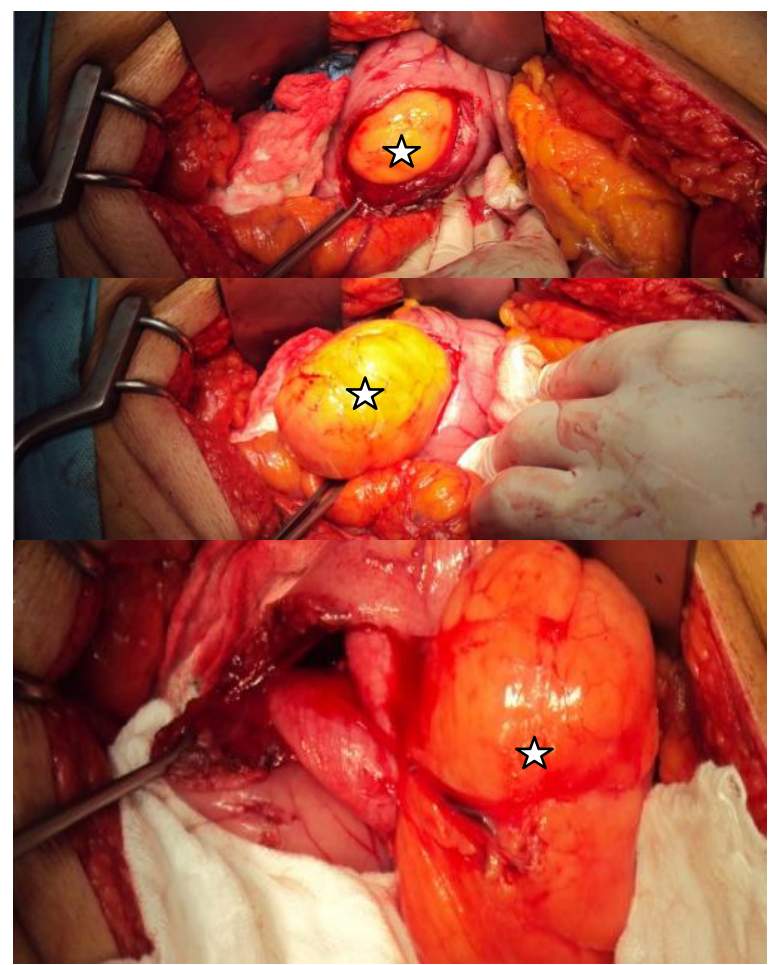

Fig. 2 Intraoperative view showing a yellowish subserosal neoplasm on the posterior wall of gastric antrum (up and middle) and the adhesion area of the gastric mucosa (bottom)

The gastrostomy was closed with PDO 3.0 in a single layer and epiploonoplasty was performed.

The pathological exam revealed that the mass is a submucosal lipoma, measuring 
$11 \times 6.5 \times 4 \mathrm{~cm}$ diameter, with areas of mucosal ulceration.

The patient underwent an uneventfull recovery and he was discharged on the seventh postoperative day.

\section{DISCUSSIONS}

Most of the gastro-intestinal lipomas can be found along the colon, ileum and jejunum. Lipomas of the esophagus and stomach represent approximately 5\% of all gastrointestinal lipomas, and when they are located in the stomach, the predilection site is the antrum. Small lipomas are usually asymptomatic, while $75 \%$ of those bigger than $4 \mathrm{~cm}$ can generate symptoms, most frequently gastrointestinal bleeding in 50\% of the patients [7], anemia, abdominal pain, dyspeptic syndrome and even obstruction [8]. In the present case, the patient presented upper gastrointestinal hemorrhage manifested by melena, dyspepsia, all due to the large size of the tumor.

To the upper gastrointestinal endoscopy, gastric lipomas appear as soft, very well defined, submucosal masses. There are 3 clues helping to identify these tumors as lipomas on endoscopy: the tenting sign (the overlying mucosa is easily retracted with the biopsy forceps), the cushion sign (the forceps produces a soft indentation on the surface of the lipoma) and naked fat sign, when fat protrudes through the overlying mucosa after multiple biopsies $[9,10]$. In the current case, the endoscopy identified the mass in the gastric antrum, but the tree signs were not present, probably due to the size of the tumor; the lesion may be associated with a central superficial ulceration [5]. The endoscopic ultrasound can be used to diagnose gastric lipomas [11] and it can identify the originating layer, the depth and the size of the tumor [12], but in our case it was not performed, for logistic reasons.

High resolution ultrasound has a detection rate of $93 \%$ in identifying submucosal masses [13]. It is difficult to do a histopatologic diagnostic after the endoscopic biopsy of these tumors, mainly because the tumors are submucosal and the obtained sample contains nonspecific tissue, such as inflammatory tissue. CT scan appears to be the most reliable diagnostic tool for ulcerative gastric lipoma [6] and this was the examination that suggested the diagnosis, due to the specific densitometry of the fatty tissue.

Lipomas are benign tumors and extensive gastric resections are not the choice in these cases [1]. The simple enucleation of the tumor or partial gastric resection have to be applied. Endoscopic polipectomy for tumors smaller than $3 \mathrm{~cm}$ gained consensus on safety and efficacy [9], while laparoscopic removal of large gastric lipomas has been successfully performed and offers advantage over an open surgery.

The therapeutic choice in this case was suggested by the imagistic diagnostic tools, especially CT-exam, but also by the intraoperative findings. The ulcerated area of the lipoma can explain the bleeding, but it can also be the consequence of the multiple biopsies. The certainty of the diagnostic was offered by the histopathological examination.

\section{CONCLUSIONS}

Gastric lipomas, depending on the size and topography, can induce a symptomatology mimicking more aggressive upper gastrointestinal tract pathology.

In most cases, lipomas are not taken into consideration as a possible differential diagnosis for the malignant tumors. Imagistic exams can be highly suggestive for a submucosal, benign tumor, while the histopathological examination of the whole resected specimen gives the final diagnosis, the endoscopic biopsies being often inconclusive.

In our case, the CT-scan suggested the diagnostic and the applied surgical treatment was the simple enucleation of the tumor.

\section{CONFLICT OF INTEREST} interest.

The authors have no conflict of 


\section{REFERENCES}

1. Salzman JR, Carr-Locke DL, Fink SA. Lipoma case report. Med Gen Med. 2005; 7(1): 16.

2. Târcoveanu E, Cotea E, Nicolescu S, et al. [Clinical significance and management in colonic lipomas]. Jurnalul de chirurgie (Iaşi). 2007; 3(4): 370-377.

3. Treska V, Pesek M, Kreuzberg B, Chudacek Z, Ludvikova M, Topolocan O. Gastric lipoma presenting as upper gastrointestinal obstruction. J Gastroenterol. 1998; 33(5): 716719.

4. Antes G, Neher M. Lipoma of the stomach diagnosis and therapy. Rontgenpraxis. 1995; 48(9): 252-253.

5. Hamdane MM, Brahim B, Belhaj Salah M, Haouas N, Bouhafa A, Chedly-Debbiche A. Giant gastric lipoma mimiking welldifferentiated liposarcoma. Gastroenterol Hepatol Bed Bench. 2012; 5(1): 60-63.

6. Thompson WM, Kende AI, Levy AD. Imaging characteristics of gastric lipomas in 16 adult and pediatric patients. Am J Roentgenol. 2003; 181(4): 981-985.
7. Ciraldo A, Thomas D, Schmidt S. Gastric lipoma presenting as gastrointetsinal bleeding : a case report. The Internet Journal of Advanced Nursing Practice. 2000; 1(1): DOI: $10.5580 / 1392$.

8. Zameer M, Kanojia RP, Rao KLN, Menon P, Samujn R, Thapa BR. Gastric lipoma. J Indian Assoc Pediatr Surg. 2010; 15(2): 64-66.

9. Taylor AJ, Stewart ET, Dodds WJ. Gastrointestinal lipomas: a radiological and pathologic view. Am J Roentgenol. 1990; 55(6): 1205-1210.

10. DeBeer RA, Shinye H. Colonic lipomas. An endoscopic analysis. Gastrointest Endosc. 1975; 22(2): 90-91.

11. Chak A. EUS in submucosal tumors. Gastrointest Endosc. 2002; 56 (4 supll): S43-S48.

12. Krasniqi AS, Hoxha FT, Bicaj BX, et al. Symptomatic subserosal gastric lipoma successfully treated with enucleation. World $J$ Gastroenterol. 2008; 14(38): 5930-5932.

13. Tsai TL, Changchien CS, Hu TH, Hsiaw CM. Demonstration of gastric submucosal lesions by high resolution transabdominal sonography. J Clin Ultrasound. 2000; 28(3): 125-132. 